\title{
Fault Identification of Rod-less Pumping with Water as Power Based on BP Neural Network
}

\author{
Lv Junyan 1, 2, a , Meng Fanzhao ${ }^{1,2, b}$, Wang Xinwei ${ }^{3, ~ c ~}$ \\ ${ }^{1}$ School of Mechanical Engineering, Shandong Huayu University of Technology, Dezhou 253034, China \\ ${ }^{2}$ Dezhou Municipal Key Laboratory of Industrial Robot Control, Dezhou 253034, China \\ ${ }^{3}$ Dezhou Community Development Center, Dezhou 253000, China \\ alvjunyan0406@126.com, bmfzhthy@163.com, ${ }^{\mathrm{c}}$ dzsgfczx@163.com
}

Keywords: fault identification, rod-less pumping, BP neural network, vibration signal

\begin{abstract}
According to the time-varying and nonlinear characteristics of rod-less pumping system with water as power, a fault identification method was put forward based on BP neural network. By studying fault mechanism and vibration signal characteristics on pumping unit, the time and frequency domain feature parameters which were used as the input of neural network were extracted. Then, according to the specific characteristics of the fault identification of pumping unit, the fault identification system was constructed. Finally, it uses the experimental data obtained by fault simulation experiment platform to construct the training samples, and train neural network based on MATLAB. The results show that BP neural network can identify the fault types of the pumping unit, which has a relatively high accuracy and feasibility.
\end{abstract}

\section{Introduction}

In recent years, China's economy is developing rapidly. The strategic position of the petroleum industry in the energy problem is more and more important. The safe and reliable production of the oil field has increasingly become the basis and premise for the construction and development of the petroleum industry [1]. Rod-less pumping with water as power is a new type of shaft production equipment, with the advantages of high pump efficiency, saving energy, reducing production cost, convenient transportation and installation, simple structure and small volume, great push the development of the application of the oil industry. Rod-less pumping with water as power is a complex mechanical system with a variable condition of continuous operation [2], the state has the characteristics of obvious time-varying nonlinear, the fault has the characteristics with concealment, complexity, uncertainty, randomness and suddenness. Artificial neural network was used in fault diagnosis in this paper, which was used to simulate the inference function of human brain so as to make fault identification [3]. The biggest feature of artificial neural network is the adaptability of the network. The network can not only learn adaptively, but also adjust the size of the network adaptively [4,5], which is very suitable for the fault identification of the rod-less pumping with water as power.

\section{The Principle of BP Neural Network}

The most widely used artificial neural network model in the fault diagnosis is the forward multi-layer neural network model, which is BP model. The main feature of BP network is the forward propagation of the signal, and the error back propagating [6]. The main structure of BP neural network is an input layer, an output layer, and a hidden layer between input layer and output layer. The hidden layer usually has one or more layers. The structure is shown in Figure 1. The neurons in the hidden layer are also called hidden units. Although the hidden layer is not directly connected to the outside world, its state directly affects the relationship between input and output [7], that is to say, changing the weight coefficient of the hidden layer can enhance the performance of the whole neural network. It is proved by theory that the three-layer forward neural network can 
approximate any continuous function with arbitrary precision [8]. In this paper, a standard threelayer neural network topology is used to form the three parts of input layer, hidden layer and output layer, which can identify the fault of pumping unit.

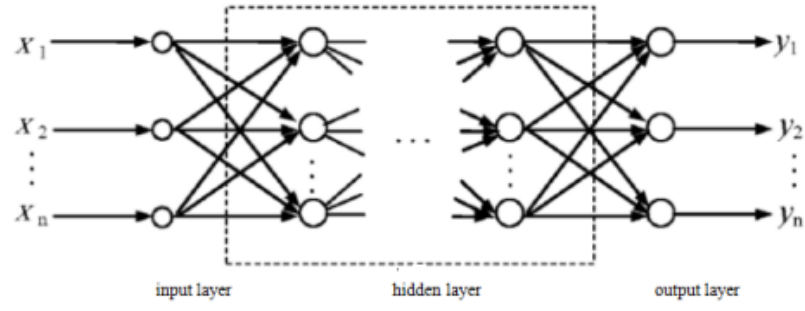

Figure 1The structure of BP neural network

BP neural network is used to train the network before it is predicted, and the network has associative memory and predictive ability through training. According to the system input and output sequence $(X, Y)$ to determine the network input layer nodes $n$, the number of hidden layer nodes $l$ and output layer nodes $m$, it initialized the input layer, hidden layer and the connection weights between the neurons in the output layer $w_{i j}, w_{j k}$, and initialized the hidden layer threshold $a$ and output layer threshold $b$, given the learning rate and the neuron activation function. The hidden layer output $H_{j}$ is:

$$
H_{j}=f\left(\sum_{i=1}^{n} w_{i j} x_{i}-a_{j}\right) j=1,2, \ldots, l
$$

In equation(1), $l$ is the number of hidden nodes, $f$ is the activation function of the hidden layer, $w_{i j}$ is the weights between the output layer and the hidden layer, $a$ is the hidden layer threshold, this function has many forms of expression. The selected function in this paper is:

$$
f(x)=\frac{1}{1+e^{-x}}
$$

This function is a more commonly used Sigmoid type function. BP neural network predicts output is $O_{k}$.

$$
O_{k}=\sum_{j=1}^{l} H_{j} w_{j k}-b_{k} k=1,2, \ldots, m
$$

Where, $H_{j}$ is the output of the hidden layer, $w_{j k}$ is the connection weight value, and $b$ is the threshold value. According to the network forecast output $O_{k}$ and expected output $Y$, it calculates the prediction error of the network $e_{k}$.

$$
e_{k}=Y_{k}-O_{k} k=1,2, \ldots, m
$$

According to the network prediction error $e_{k}$ to update the network connection weights $w_{i j}, w_{j k}$.

$$
\begin{aligned}
& w_{i j}=w_{i j}+\eta H_{j}\left(1-H_{j}\right) x(i) \sum_{k=1}^{m} w_{j k} e_{k} i=1,2, \ldots, n ; j=1,2, \ldots, l \\
& w_{j k}=w_{j k}+\eta H_{j} e_{k} j=1,2, \ldots, l ; k=1,2, \ldots, m
\end{aligned}
$$

Where $\eta$ is the learning rate.

\section{Example Verification}

In the paper, BP neural network is used to identify the pumping units. The modeling of the 
algorithm includes three parts: the construction of BP neural network, the training of BP neural network and the identification of BP neural network. It carried out de-noising the collected data, and then calculated the 20 groups characteristic parameter, including 13 time-domain features and 7 frequency domain features. Classify the 7 types of characteristic signals as one group, and then randomly select 1500 groups as training data, 300 groups as test data, and normalize the training data. Set the desired output of each group of signals according to the category of the signal, for example, when the signal is 1, the desired output vector is $\left[\begin{array}{lllllll}1 & 0 & 0 & 0 & 0 & 0 & 0\end{array}\right]$. Because the input signal consists of 21 dimensions, there are 7 kinds of signals to be diagnosed, so the structure of BP neural network is, the input layer has 21 nodes, the hidden layer has 15 nodes, and the output layer has 7 nodes. The BP neural network training consists of two parts: the initialization of the BP neural network and the training of the BP neural network. The 7 sets of data are made up of one group and then divided into 1800 groups. It selects 1500 groups randomly as training data for training the network, and the remaining 300 groups are used to test the recognition ability of the network.

\section{Diagnostic Results and Experimental Analysis}

The trained BP neural network is used to identify the test data, and the BP neural network diagnosis error is shown in Figure 2 and Figure 3.

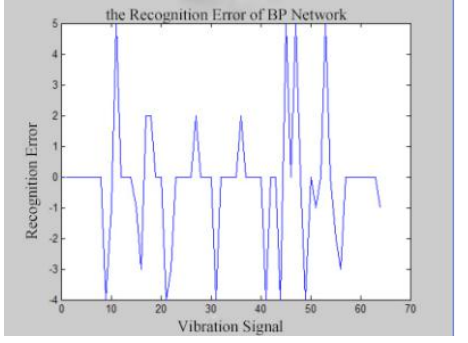

Figure 2 The identification error

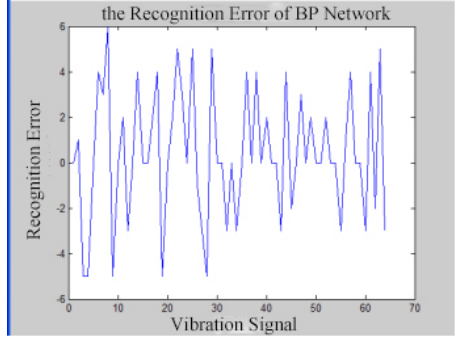

Figure 3 The identification error

It can be seen that the number of nodes in the hidden layer of the BP neural network has a great influence on the diagnostic accuracy of the BP neural network. Too few nodes will lead neural network not to be trained and learned well. It requires the increase of the training times, also will affect the precision of training; too many nodes, the training time will increase, learning the details of the training network will be too much, leading to over-fitting phenomenon. The optimal number of hidden layer nodes can be determined by the following equation:

$$
\left.\begin{array}{c}
l<n-1 \\
l=\log _{2} n \\
l<\sqrt{(m+n)}+a
\end{array}\right\}
$$

Table 1 The recognition rate of BP network

\begin{tabular}{|c|c|}
\hline Vibration Signal Recognition & Recognition Accuracy \\
\hline First kind & 0.9333 \\
\hline Second kind & 0.9778 \\
\hline Third kind & 1 \\
\hline Forth kind & 0.9392 \\
\hline Fifth kind & 0.9743 \\
\hline Sixth kind & 0.9364 \\
\hline Seventh kind & 0.9578 \\
\hline
\end{tabular}

Where, $n$ is the number of nodes in the input layer; $l$ is the number of nodes in the hidden layer; $m$ is the number of nodes in the output layer; $a$ is the constant between 0 and 10. In practical problems, the number of nodes in the hidden layer is to determine the approximate range by the above equation, and then determine the number of the most suitable nodes by the trial and error method. Figure 2 shows the fault identification error when the number of hidden layer nodes is 15, Figure 3 
shows the identification error when the number of hidden layer nodes is 6 , from which it can be seen that when the number of nodes in the hidden layer is 15, the fault identification error of pumping unit is minimum, i.e. when the hidden layer nodes the number is 15 , the effect of system identification is the best. When the number of hidden layer nodes is 15 , the identification accuracy of the BP neural network is shown in Table 1.

Seen from Table1, the vibration signal based on BP neural network algorithm has high accuracy, which can accurately identify the fault type of the vibration signal, prove that the BP neural network is very accurate and feasible for the fault identification of the rod-less pumping with water as power.

\section{Conclusions}

Through the training of the network by using the physical fault data, it is proved that the network can identify the fault of the rod-less pumping with water as power with the high accuracy, which can provide help for the operation and maintenance of the pumping unit. Compared with the manifold learning methods for fault identification, the method in the paper directly illustrates the results with the correct recognition rate, and is compared to the manifold image observation method, BP neural network makes the identification simpler, which is fully applicable to the fault identification of the rod-less pumping with water as power, and has broad prospects for development.

\section{References}

[1] Shan Meichen. (2013) Research on fault diagnosis method for the rod-less pumping with water as power[D]. BeiJing: BeiJing Information Science \&Technology University.

[2] Chen Xiangzhen.(2014) Analysis about the Influencing Factors of the Working Efficiency of the Pumping Unit Based on the Orthogonal Test[J]. China Petroleum Machinery, 42,61-64

[3] Yu Tingting.(2008) Fault Diagnosis Method of Rolling Bearing Based on BP Neural Network [D]. DaLian: DaLian University of Technology.

[4] Xu Ning, Huang Zhichu.(2005) Application of Neural Network in Fault Diagnosis of Rotating Machinery [J]. Mining Machinery,8,86-87.

[5] B. Li, Y. Tipsuwan. (2000) Neural-network-based motor rolling bearing fault diagnosis[J]. Industrial Electronics,47,1060-1069.

[6] Wang Xiaochuan, Shi Feng.(2014) 43 Case Analysis of MATLAB Neural Network [M]. BeiJing: Beihang University Press.

[7] Zhang Wenge, Wu Zening.(2003) Improvement and Application of BP Neural Network[J]. Henan University.2,21-26.

[8] Qi Yong, Li Qianmu.(2004) Network Intelligent Diagnosis System Based on BP Neural Network [J]. Microellectronics \& Computer,21,10-13. 